K. SEKIGAWA KODAI MATH: J. € (1983), 174-185

\title{
ALMOST COMPLEX SUBMANIFOLDS OF A 6-DIMENSIONAL SPHERE
}

\author{
By Kouei Sekigawa
}

\section{Introduction}

Among all submanifolds of an almost Hermitian manifold, there are two typical classes: one is the class of almost complex submanifolds, and the other is the class of totally real submanifolds. A Riemannian submanifold $(M, \phi)$ (or briefly $M$ ) of an almost Hermitian manifold $(\tilde{M}, J,\langle$,$\rangle ) (or briefly \tilde{M}$ ) is called an almost complex submanifold provided that $J_{\psi(p)}\left((d \psi)_{p}(X)\right) \in(d \psi)_{p}\left(T_{p}(M)\right)$ for any $X \in T_{p}(M), p \in M$. The most typical example of nearly Kaehlerian manifolds is a 6-dimensional sphere $S^{6}$. In fact, Fukami and Ishihara [3] proved that there exists a nearly Kaehlerian structure on a 6-dimensional sphere $S^{6}$ by making use of the properties of the Cayley division algebra. We shall call it the canonical nearly Kaehlerian structure on $S^{6}$. In this paper, we shall study almost complex submanifolds of a 6-dimensional unit sphere $S^{6}$ with the canonical nearly Kaehlerian structure. First of all, Gray [1] proved that with respect to the canonical nearly Kaehlerian structure, $S^{6}$ has no 4-dimensional almost complex submanifolds. We shall prove the following Theorems and some related results. In the following Theorems, we assume that $M=(M, \phi)$ is an almost complex submanifold of $S^{6}$. Then it follows that $\operatorname{dim} M=2$. We denote by $K$ the Gaussian curvature of $M$.

THEOREm A. If $(M, \psi)$ is not totally geodesic, then the degree of $\psi$ is 3 .

Theorem B. If $K$ is constant on $M$, then $K=1$ or $1 / 6$ or 0 .

Theorem C. Assume that $M$ is compact. If $K>1 / 6$ on $M$, then $K=1$ on $M$, and if $1 / 6 \leqq K<1$ on $M$, then $K=1 / 6$ on $M$.

In the last section of this paper, we shall give some examples of almost complex submanifolds of $S^{6}$ corresponding to the cases, $K=1,1 / 6$ and 0 in Theorem B. We note that the result of Theorem B is a special case of the result obtained by Kenmotsu under more general situation ([6]).

Received July 8, 1982 


\section{Riemannian submanifolds}

Let $(\tilde{M},\langle\rangle$,$) (or briefly \tilde{M}$ ) be a Riemannian manifold and $(M, \psi)$ (or brieflv $M)$ be a Riemannian submanifold of $\tilde{M}$ with isometric immersion $\psi$. Let $\nabla$ (resp. $\tilde{\nabla})$ be the Riemannian connection on $M$ (resp. $\tilde{M}$ ) and $R$ (resp. $\tilde{R}$ ) be the curvature tensor of $M$ (resp. $\tilde{M})$. We denote by $\sigma$ the second fundamental form of $M$ in $\tilde{M}$. Since $\phi$ is locally an imbedding, we may identify $p \in M$ with $\phi(p) \in \tilde{M}$ locally, and $T_{p}(M)$ with the subspace $(d \psi)_{p}\left(T_{p}(M)\right)$ of $T_{\psi(p)}(\tilde{M})$. Then, the Gauss formula, Weingarten formula are given respectively by

$$
\begin{gathered}
\sigma(X, Y)=\tilde{\nabla}_{X} Y-\nabla_{X} Y, \\
\tilde{\nabla}_{X} \xi=-A_{\xi} X+\nabla_{X}^{\perp} \xi, \quad X, Y \in \mathfrak{X}(M),
\end{gathered}
$$

where $\xi$ is a local field of normal vector to $M$ and $-A_{\xi} X$ (resp. $\nabla_{\bar{X}}^{\frac{1}{X}} \xi$ ) denotes the tangential part (resp. normal part) of $\tilde{\nabla}_{X} \xi$.

The tangential part $A_{\xi} X$ is related to the second fundamental form $\sigma$ as follows :

$$
\langle\sigma(X, Y), \xi\rangle=\left\langle A_{\xi} X, Y\right\rangle, \quad X, Y \in \mathfrak{X}(M) .
$$

We denote by $R^{\perp}$ the curvature tensor of the normal connection, i.e., $R^{\perp}(X, Y)=$ $\left[\nabla_{X}^{\perp}, \nabla_{Y}^{\perp}\right]-\nabla_{[X, Y]}^{\perp}$. Then, the Gauss, Codazzi and Ricci equations are given respectively by

$$
\begin{gathered}
\left\langle R(X, Y) Z, Z^{\prime}\right\rangle=\left\langle\tilde{R}(X, Y) Z, Z^{\prime}\right\rangle+\left\langle\sigma\left(X, Z^{\prime}\right), \sigma(Y, Z)\right\rangle \\
-\left\langle\sigma(X, Z), \sigma\left(Y, Z^{\prime}\right)\right\rangle, \\
(\tilde{R}(X, Y) Z)^{\perp}=\left(\nabla_{X}^{\prime} \sigma\right)(Y, Z)-\left(\nabla_{Y}^{\prime} \sigma\right)(X, Z), \\
\langle\tilde{R}(X, Y) \xi, \eta\rangle=\left\langle R^{\perp}(X, Y) \xi, \eta\right\rangle-\left\langle\left[A_{\xi}, A_{\eta}\right] X, Y\right\rangle,
\end{gathered}
$$

for $X, Y, Z, Z^{\prime} \in \mathfrak{X}(M)$, where $\left(\nabla_{X}^{\prime} \sigma\right)(Y, Z)=\nabla_{X}^{\perp} \sigma(Y, Z)-\sigma\left(\nabla_{X} Y, Z\right)-\sigma\left(Y, \nabla_{X} Z\right)$ and $\xi, \eta$ are local fields of normal vectors to $M$.

In the sequel, the following convention for the notations will be used unless otherwise specified:

$$
X, Y, Z, \cdots, \in \mathfrak{X}(M), \quad U, V, W, \cdots, \in \mathfrak{X}(\tilde{M})
$$

and $\mathfrak{X}(M)$ (resp. $\mathfrak{X}(\tilde{M})$ ) denotes the set of all tangential vector fields to $M$ (resp. $\tilde{M})$. [8].

For the definition of the degree of the isometric immersion $\psi$, we refere to 


\section{6-dimensional nearly Kaehlerian manifolds}

In this section, for the sake of later uses, we shall recall some elementary formulas in a 6-dimensional nearly Kaehlerian manifold and furthermore the canonical nearly Kaehlerian structure on a 6 -dimensional unit sphere $S^{6}$. Let $\tilde{M}$ be an almost Hermitian manifold with the almost Hermitian structure $(J,\langle\rangle$,$) .$ We denote by $N$ the Nijienhuis tensor of $J$ and by $\tilde{\nabla}$ the Riemannian connection of $\tilde{M}$. It is known that the tensor field $N$ satisfies

$$
N(J U, V)=N(U, J V)=-J N(U, V), \quad U, V \in \mathfrak{X}(\tilde{M}) .
$$

Especially, if $\tilde{M}$ is a nearly Kaehlerian manifold (i.e., $\left(\tilde{\nabla}_{U} J\right) U=0$, for any $U \in \mathfrak{X}(\tilde{M})$, then the tensor field $N$ is written in the following form (cf. [13]):

$$
N(U, V)=-4 J\left(\tilde{\nabla}_{U} J\right) V, \quad U, V \in \mathfrak{X}(\tilde{M}) .
$$

From (3.2), we get

$$
\langle N(U, V), W\rangle=-\langle N(U, W), V\rangle, \quad U, V, W \in \mathfrak{X}(\tilde{M}) .
$$

An almost complex submanifold $M$ of an almost Hermitian manifold $\tilde{M}$ is called to be a $\sigma$-submanifold if the second fundamental form $\sigma$ is complex linear, i.e.,

$$
\sigma(J X, Y)=\sigma(X, J Y)=J \sigma(X, Y), \quad \text { for } \quad X, Y \in \mathfrak{X}(M),
$$

(cf. [12]). From (3.4), any $\sigma$-submanifold is necessarily minimal. Vanhecke [12] proved that if $\tilde{M}$ is a nearly Kaehlerian manifold, any almost complex submanifold is a $\sigma$-submanifold and is also a nearly Kaehlerian manifold. W now assume that $\tilde{M}$ is a 6 -dimensional non-Kaehlerian, nearly Kaehlerian manifold. Then the followings hold in $\tilde{M}$ (cf. [7], [9]):

$$
\begin{gathered}
\tilde{\nabla}_{U}\left(\left(\tilde{\nabla}_{V} J\right) W\right)-\left(\tilde{\nabla}_{\tilde{\nabla}_{U}} J\right) W-\left\langle\tilde{\nabla}_{V} J\right)\left(\tilde{\nabla}_{U} W\right) \\
=-\frac{S}{30}(\langle U, V\rangle J W-\langle U, W\rangle J V+\langle J V, W\rangle U), \\
\left(\tilde{\nabla}_{U} J\right)\left(\tilde{\nabla}_{V} J\right) W=-\frac{S}{30}(\langle U, V\rangle W-\langle U, W\rangle V \\
+\langle J U, V\rangle J W-\langle J U, W\rangle J V),
\end{gathered}
$$

$U, V, W \in \mathfrak{X}(\tilde{M})$, where $S$ denotes the scalar curvature of $\tilde{M}$.

From (3.2), (3.5) and (3.6), we get

$$
\begin{aligned}
& \left(\tilde{\nabla}_{U} N\right)(V, W)=\frac{2 S}{15}(\langle J U, V\rangle J W-\langle J U, W\rangle J V+\langle J V, W\rangle J U, \\
& N(U, N(V, W))=16\left(\tilde{\nabla}_{U} J\right)\left(\tilde{\nabla}_{V} J\right) W
\end{aligned}
$$




$$
=-\frac{8 S}{15}(\langle U, V\rangle W-\langle U, W\rangle V+\langle J U, V\rangle J W-\langle J U, W\rangle J V),
$$

$$
\begin{aligned}
& \left\langle N(U, V), N\left(U^{\prime}, V^{\prime}\right)\right\rangle=-16\left\langle V,\left(\tilde{\nabla}_{U} J\right)\left(\tilde{\nabla}_{U^{\prime}} J\right) V^{\prime}\right\rangle \\
& =\frac{8 S}{15}\left(\left\langle U, U^{\prime}\right\rangle\left\langle V, V^{\prime}\right\rangle-\left\langle U, V^{\prime}\right\rangle\left\langle V, U^{\prime}\right\rangle\right. \\
& \left.\quad+\left\langle J U, U^{\prime}\right\rangle\left\langle J V^{\prime}, V\right\rangle-\left\langle J U, V^{\prime}\right\rangle\left\langle J U^{\prime}, V\right\rangle\right),
\end{aligned}
$$

$U, U^{\prime}, V, V^{\prime}, W \in \mathfrak{X}(\tilde{M})$.

We shall now recall the canonical nearly Kaehlerian structure on a 6-dimensional sphere $S^{6}$. Let $\mathcal{C}$ be the Cayley division algebra generated by $\left\{e_{0}=1\right.$, $\left.e_{2}(1 \leqq \imath \leqq 7)\right\}$ over real number field $\boldsymbol{R}$ and $\mathcal{C}_{+}$be the subspace of $\mathcal{C}$ consisting of all purely imaginary Cayley numbers. We may identify $\mathcal{C}_{+}$with a 7 -dimensional Euclidean space $\boldsymbol{R}^{7}$ with the canonical inner product (,) (i.e., $\left(e_{\imath}, e_{\jmath}\right)=\delta_{i \jmath}, 1 \leqq \imath$, $j \leqq 7$ ). The automorphism group of $\mathcal{C}$ is the compact simple Lie group $G_{2}$ and the inner product $($,$) is invariant under the action of the group G_{2}$. A vector cross product for the vectors in $\mathcal{C}_{+}=\boldsymbol{R}^{7}$ is defined by

$$
x \times y=(x, y) e_{0}+x y, \quad x, y \in \mathcal{C}_{+} .
$$

Then the multiplication table is given by the following:

$$
\begin{array}{r|rrrrrrr}
\hline k & 1 & 2 & 3 & 4 & 5 & 6 & 7 \\
\hline j & \multicolumn{1}{c}{0} & e_{3} & -e_{2} & e_{5} & -e_{4} & e_{7} & -e_{6} \\
2 & -e_{3} & 0 & e_{1} & e_{6} & -e_{7} & -e_{4} & e_{5} \\
3 & e_{2} & -e_{1} & 0 & -e_{7} & -e_{6} & e_{5} & e_{4} \\
4 & -e_{5} & -e_{6} & e_{7} & 0 & e_{1} & e_{2} & -e_{3} \\
5 & e_{4} & e_{7} & e_{6} & -e_{1} & 0 & -e_{3} & -e_{2} \\
6 & -e_{7} & e_{4} & -e_{5} & -e_{2} & e_{3} & 0 & e_{1} \\
7 & e_{6} & -e_{5} & -e_{4} & e_{3} & e_{2} & -e_{1} & 0 \\
\hline
\end{array}
$$

Considering $S^{6}$ as $\left\{x \in \mathcal{C}_{+} ;(x, x)=1\right\}$, the canonical almost complex structure $J$ on $S^{6}$ is defined by

$$
J_{x} U=x \times U,
$$

where $x \in S^{6}$ and $U \in T_{x}\left(S^{6}\right)$ (the tangent space of $S^{6}$ at $x$ ).

The above almost complex structure $J$ together with the induced Riemannian metric $\langle$,$\rangle on S^{6}$ from the inner product (, ) on $\mathcal{C}_{+}=\boldsymbol{R}^{7}$ gives rise to a nearly Kaehlerian structure on $S^{6}$. The group $G_{2}$ acts on $S^{6}$ transitively as the group of automorphisms of the nearly Kaehlerian structure $(J,\langle\rangle$,$) (cf. [3]). It is$ well known that $S^{6}$ does not admit any Kaehlerian structures. 


\section{Proofs of Theorems A, B and C}

Let $M$ be an almost complex submanifold of a 6-dimensional unit sphere $\tilde{M}=S^{6}$ with the canonical nearly Kaehlerian structure $(J,\langle\rangle$,$) . Then it follows$ that $\operatorname{dim} M=2$ and hence $M$ is a Kaehlerian manifold of complex dimension 1 with respect to the induced structure from $S^{6}$. We denote by $K$ the Gaussian curvature of $M$. Then, from (2.4) and (3.4), we get

$$
K=1-\frac{\|\sigma\|^{2}}{2}
$$

where $\|\sigma\|$ denotes the length of the second fundamental form $\sigma$.

Codazzi equation (2.5) implies in particular

$$
\left(\nabla_{X}^{\prime} \sigma\right)(Y, Z)=\left(\nabla_{Y}^{\prime} \sigma\right)(X, Z) .
$$

From (2.1), (2.2) and (3.2), we get

$$
\begin{aligned}
& \tilde{\nabla}_{Z}(J \sigma(X, Y))=\frac{1}{4} J N(Z, \sigma(X, Y))+J\left(-A_{\sigma(X, Y)} Z+\nabla_{\frac{1}{Z}} \sigma(X, Y)\right), \\
& \tilde{\nabla}_{Z}(\sigma(X, J Y))=-A_{\sigma(X, J Y)} Z+\nabla_{Z}^{\frac{1}{Z}} \sigma(X, J Y) .
\end{aligned}
$$

From (4.3), taking account of (3.1), (3.3) and (3.4), we get

$$
\frac{1}{4} J N(Z, \sigma(X, Y))=\left(\nabla_{Z}^{\prime} \sigma\right)(X, J Y)-J\left(\nabla_{Z}^{\prime} \sigma\right)(X, Y) .
$$

Since $\operatorname{dim} M=2$, from (3.1) and (3.4), we get easily

$$
N(Z, \sigma(X, Y))=N(Y, \sigma(X, Z)) .
$$

Let $M^{\prime}=\{p \in M ; \sigma \neq 0$ at $p\}$. Then $M^{\prime}$ is an open set of $M$.

We now assume that $M^{\prime} \neq \emptyset$ (i.e., $M$ is not totally geodesic in $S^{6}$ ). Let $\left\{X_{1}, X_{2}=J X_{1}\right\}$ be a local field of orthonormal frame on a neighborhood of a point $p \in M^{\prime}$ in $M$. If we put

$$
\nabla_{X_{\imath}} X_{\jmath}=\sum_{k=1}^{2} B_{\imath j k} X_{k}, \quad 1 \leqq i, \jmath \leqq 2,
$$

then we get

$$
B_{\imath \jmath k}=-B_{\imath k \jmath}, \quad 1 \leqq i, \jmath, k \leqq 2 .
$$

Taking account of (3.1), (3.3), (3.4) and (3.9), we may put

$$
\begin{aligned}
\left(\nabla_{X_{1}}^{\prime} \sigma\right)\left(X_{1}, X_{1}\right)= & a \sigma\left(X_{1}, X_{1}\right)+b \sigma\left(X_{1}, X_{2}\right) \\
& +\frac{c}{4} N\left(X_{1}, \sigma\left(X_{1}, X_{1}\right)\right)+\frac{d}{4} N\left(X_{2}, \sigma\left(X_{1}, X_{1}\right)\right),
\end{aligned}
$$




$$
\begin{aligned}
\left(\nabla_{X 2}^{\prime} \sigma\right)\left(X_{1}, X_{1}\right)= & a^{\prime} \sigma\left(X_{1}, X_{1}\right)+b^{\prime} \sigma\left(X_{1}, X_{2}\right) \\
& +\frac{c^{\prime}}{4} N\left(X_{1}, \sigma\left(X_{1}, X_{1}\right)\right)+\frac{d^{\prime}}{4} N\left(X_{2}, \sigma\left(X_{1}, X_{1}\right)\right) .
\end{aligned}
$$

Then, from (4.8), taking account of (2.5), (3.1), (3.4) and (4.4), we get

$$
a^{\prime}=-b, \quad b^{\prime}=a, \quad c^{\prime}=d, \quad d^{\prime}=-c-1 .
$$

Thus, from (4.8), taking account of (3.3), (3.4) and (4.9), we get

$$
a=\frac{1}{\|\sigma\|} X_{1}\|\sigma\|, \quad b=-\frac{1}{\|\sigma\|} X_{2}\|\sigma\| .
$$

From (4.6), (4.7) and (4.10), we get

$$
\begin{aligned}
{\left[X_{1}, X_{2}\right]\|\sigma\| } & =X_{1}\left(X_{2}\|\sigma\|\right)-X_{2}\left(X_{1}\|\sigma\|\right) \\
& =-X_{1}(b\|\sigma\|)-X_{2}(a\|\sigma\|) \\
& =-\left(X_{1} b+X_{2} a\right)\|\sigma\|,
\end{aligned}
$$

and hence

$$
X_{2} a+X_{1} b+a B_{121}+b B_{212}=0 .
$$

Taking account of (3.4), (4.6) and (4.7), we get easily

$$
\sum_{\imath=1}^{2}\left(\nabla_{X}^{\prime} \sigma\right)\left(X_{\imath}, X_{\imath}\right)=0
$$

From (4.8) with (4.9), taking account of (2.5), (3.1), (3.3) (3.6) and (4.12), we get

$$
\begin{aligned}
\left\|\nabla^{\prime} \sigma\right\|^{2}= & \sum_{1 \leqq 2, \jmath, k \leqq 2}\left\langle\left(\nabla_{X_{i}}^{\prime} \sigma\right)\left(X_{\jmath}, X_{k}\right),\left(\nabla_{X_{i}}^{\prime} \sigma\right)\left(X_{\jmath}, X_{k}\right)\right\rangle \\
= & 4\left(\left\langle\left(\nabla_{X_{1}}^{\prime} \sigma\right)\left(X_{1}, X_{1}\right),\left(\nabla_{X_{1}}^{\prime} \sigma\right)\left(X_{1}, X_{1}\right)\right\rangle\right. \\
& \left.+\left\langle\left(\nabla_{X_{2}}^{\prime} \sigma\right)\left(X_{1}, X_{1}\right),\left(\nabla_{X_{2}}^{\prime} \sigma\right)\left(X_{1}, X_{1}\right)\right\rangle\right) \\
= & \left(2\left(a^{2}+b^{2}\right)+2\left(c^{2}+c+d^{2}\right)+1\right)\|\sigma\|^{2} .
\end{aligned}
$$

From (4.10) and (4.13), we get

$$
\begin{aligned}
& a^{2}+b^{2}=\|\operatorname{grad}(\log \|\sigma\|)\|^{2}, \\
& c^{2}+c+d^{2}=\frac{1}{2\|\sigma\|^{2}}-\left(\left\|\nabla^{\prime} \sigma\right\|^{2}-2\|\operatorname{grad}\| \sigma\|\|^{2}-\|\sigma\|^{2}\right) .
\end{aligned}
$$

We put

$$
F=\|\operatorname{grad}(\log \|\sigma\|)\|^{2}
$$

and

$$
G=\frac{1}{2\|\sigma\|^{2}}\left(\left\|\nabla^{\prime} \sigma\right\|^{2}-2\|\operatorname{grad}\| \sigma\|\|^{2}-\|\sigma\|^{2}\right)
$$


Then, from (4.15), we have easily

LEMMA 4.1. $G \geqq-\frac{1}{4}$ on $M^{\prime}$.

From (2.6), taking account of (2.1), (2,2), (3.1) (3.4), (3.7), (3.8), (4.1), (4.5) (4.9), we get

$$
\begin{aligned}
-\frac{1}{8}\|\sigma\|^{4}= & \left\langle R^{\perp}\left(X_{1}, X_{2}\right) \sigma\left(X_{1}, X_{1}\right), \sigma\left(X_{1}, X_{2}\right)\right\rangle \\
= & \frac{\|\sigma\|^{2}}{4}\left(X_{1} a-X_{2} b-b B_{121}+a B_{212}-2 G-1\right. \\
& \left.+2\left(X_{1} B_{212}-X_{2} B_{112}-B_{121} B_{112}+B_{212} B_{212}\right)\right) \\
= & \frac{\|\sigma\|^{2}}{4}\left(X_{1} a-X_{2} b-b B_{121}+a B_{212}-1-2 G-2 K\right),
\end{aligned}
$$

and hence

$$
X_{1} a-X_{2} b-b B_{121}+a B_{212}=2 G+3 K \text {. }
$$

Similarly, we get

$$
\begin{aligned}
& X_{1} d-X_{2} c=3(2 c+1) B_{121}-6 d B_{212}-2 a d-(2 c+1) b, \\
& X_{1} c+X_{2} d=-6 d B_{121}-3(2 c+1) B_{212}+2 b d-(2 c+1) a .
\end{aligned}
$$

LEMMA 4.2. $\Delta(\log \|\sigma\|)=2 G+3 K$ on $M^{\prime}$.

Proof. From (4.6), (4.7), (4.10) and (4.16), we get

$$
\begin{aligned}
\Delta\|\sigma\| & =X_{1}\left(X_{1}\|\sigma\|\right)+X_{2}\left(X_{2}\|\sigma\|\right)+B_{121} X_{2}\|\sigma\|+B_{212} X_{1}\|\sigma\| \\
& =\|\sigma\|\left(X_{1} a-X_{2} b-b B_{121}+a B_{212}+a^{2}+b^{2}\right) \\
& =\|\sigma\|(F+2 G+3 K)
\end{aligned}
$$

and hence

$$
\begin{aligned}
\Delta(\log \|\sigma\|) & =(1 /\|\sigma\|) \Delta\|\sigma\|-\|\operatorname{grad}(\log \|\sigma\|)\|^{2} \\
& =2 G+3 K
\end{aligned}
$$

Let $\left\{E_{1}, E_{2}=J E_{1}\right\}$ be an orthonormal basis of $T_{p}(M), \quad p \in M^{\prime}$ and $\gamma_{\imath}=\gamma_{\imath}\left(t_{2}\right)$ $(1 \leqq i \leqq 2)$ be the geodesics in $M^{\prime}$ such that

$$
\gamma_{i}(0)=p \quad \text { and } \quad \frac{d \gamma_{\imath}}{d t_{\imath}}(0)=E_{\imath}, \quad 1 \leqq i \leqq 2 .
$$

Then, we may easily see that there exists an orthonormal frame field $\left\{X_{1}, X_{2}=\right.$ $\left.J X_{1}\right\}$ near $D$ in $M^{\prime}$ such that 


$$
X_{\imath}=E_{\imath} \quad\left(1 \leqq_{\imath} \leqq 2\right) \quad \text { at } p,
$$

and

$$
X_{1}=\frac{d \gamma_{1}}{d t_{1}} \text { along } \gamma_{1}, \quad X_{2}=\frac{d \gamma_{2}}{d t_{2}} \text { along } \gamma_{2}
$$

From (4.19), we get

$$
B_{121}=0 \text { along } \gamma_{1} \text { and } B_{212}=0 \text { along } \gamma_{2} \text {. }
$$

From (4.17) and (4.18), taking account of (4.19) and (4.20), we get

$$
\begin{aligned}
E_{1}\left(X_{1} d\right)-E_{1}\left(X_{2} c\right)= & -(2 c+1) E_{1} d-2 d E_{1} a \\
& -6 d E_{1} B_{212}-2 b E_{1} c-2 a E_{1} d, \\
E_{2}\left(X_{1} c\right)+E_{2}\left(X_{2} d\right)= & -(2 c+1) E_{2} a+a d E_{2} b \\
& -6 d E_{2} B_{121}-2 a E_{2} c+2 b E_{2} d .
\end{aligned}
$$

From (4.21), taking account of (4.11), (4.16) and (4.20), we get

$$
d=-4 d G-2 a E_{1} d+2 b E_{2} d-2 b E_{1} c-2 a E_{2} c .
$$

Similarly, we get

$$
c=-2(2 c+1) G+2 b E_{1} d+2 a E_{2} d-2 a E_{1} c+2 b E_{2} c .
$$

On one hand, from (4.17), (4.18) and (4.20), we get

$$
\begin{aligned}
& \left(E_{1} c\right)^{2}=-\left(E_{1} c\right)\left(E_{2} d\right)-(2 c+1) a E_{1} c+2 b d E_{1} c, \\
& \left(E_{2} c\right)^{2}=\left(E_{2} c\right)\left(E_{1} d\right)+2 a d E_{2} c+(2 c+1) b E_{2} c, \\
& \left(E_{1} d\right)^{2}=\left(E_{2} c\right)\left(E_{1} d\right)-2 a d E_{1} d-(2 c+1) b E_{1} d, \\
& \left(E_{2} d\right)^{2}=-\left(E_{1} c\right)\left(E_{2} d\right)-(2 c+1) a E_{2} d+2 b d E_{2} d .
\end{aligned}
$$

From (4.17), (4.18) and (4.24), we get

$$
\begin{aligned}
& 2\left(\left(E_{2} c\right)\left(E_{1} d\right)-\left(E_{1} c\right)\left(E_{2} d\right)\right) \\
& \quad=-F(4 G+1)+\left(E_{1} c\right)^{2}+\left(E_{2} c\right)^{2}+\left(E_{1} d\right)^{2}+\left(E_{2} d\right)^{2} .
\end{aligned}
$$

Thus, from $(4.21) \sim(4.25)$, we get

$$
\begin{aligned}
\Delta G= & 2\left(-(F+G)(4 G+1)-2 a E_{1} G+2 b E_{2} G\right. \\
& \left.+\left(E_{1} c\right)^{2}+\left(E_{2} c\right)^{2}+\left(E_{1} d\right)^{2}+\left(E_{2} d\right)^{2}\right) .
\end{aligned}
$$

LEMMA 3. The following holds on $M^{\prime}$.

$$
\Delta(4 G+1)^{3}=24(4 G+1)\left(-(4 G+1)^{2} G+6\left\|\operatorname{grad} G^{\prime 2}\right\|^{2} .\right.
$$


Proof. By the definition of the function $G$, we get

$$
E_{i} G=(2 c+1) E_{i} c+2 d E_{i} d, \quad 1 \leqq i \leqq 2 .
$$

From (4.17), (4.18) and (4.28), we get

$$
\begin{aligned}
& (4 G+1) E_{1} c=(2 c+1) E_{1} G-2 d E_{2} G+2 b d(4 G+1), \\
& (4 G+1) E_{2} c=2 d E_{1} G+(2 c+1) E_{2} G+2 a d(4 G+1), \\
& (4 G+1) E_{1} d=2 d E_{1} G+(2 c+1) E_{2} G-(2 c+1) b(4 G+1), \\
& (4 G+1) E_{2} d=-(2 c+1) E_{1} G+2 d E_{2} G-(2 c+1) a(4 G+1) .
\end{aligned}
$$

From (4.29), taking account of the definitions of the functions $F$ and $G$, we get

$$
\begin{gathered}
(4 G+1)^{2}\left(\left(E_{1} c\right)^{2}+\left(E_{2} c\right)^{2}+\left(E_{1} d\right)^{2}+\left(E_{2} d\right)^{2}\right) \\
=2(4 G+1)\left((4 G+1)^{2} F+\|\operatorname{grad} G\|^{2}\right. \\
\left.+a(4 G+1) E_{1} G-b(4 G+1) E_{2} G\right) .
\end{gathered}
$$

Thus, from (4.26) and (4.30), we have finally (4.27).

Q.E.D.

We are now in a position to prove Theorems A, B and C. First, we shall prove Theorem A. We denote by $\nu_{p}^{k}$ the $k$-th normal space and by $\sigma_{p}^{k}$ the $k$-th fundamental form of the isometric immersion $\phi$ at $p \in M^{\prime}$. Then from (4.8) with (4.9), we see that $\nu_{p}^{1}$ and $\nu_{p}^{2}$ are generated respectively by $\left\{\sigma_{p}^{2}\left(E_{1}, E_{1}\right)=\sigma\left(E_{1}, E_{1}\right)\right.$, $\left.\sigma_{p}^{2}\left(E_{1}, E_{2}\right)=\sigma\left(E_{1}, E_{2}\right)\right\}$ and $\left\{\sigma_{p}^{3}\left(E_{1}, E_{1}, E_{1}\right)=(c / 4) N\left(E_{1}, \sigma\left(E_{1}, E_{1}\right)\right)+(d / 4) N\left(E_{2}\right.\right.$, $\left.\left.\sigma\left(E_{1}, E_{1}\right)\right), \sigma_{p}^{3}\left(E_{2}, E_{1}, E_{1}\right)=(d / 4) N\left(E_{1}, \sigma\left(E_{1}, E_{1}\right)\right)-((c+1) / 4) N\left(E_{2}, \sigma\left(E_{1}, E_{1}\right)\right)\right\}$, where $E_{2}=J E_{1}$.

If $G(p) \neq 0$, then it follows that $\operatorname{dim} \nu_{p}^{1}=2, \operatorname{dim} \nu_{p}^{2}=2$, and hence the degree of the immersion $\phi$ is 3. So, we assume that $G=0$ on $M^{\prime}$. Let $p$ be any point of $M^{\prime}$ and define $E$ by

$$
\left\|\left(\nabla_{E}^{\prime} \sigma\right)(E, E)\right\|=\operatorname{Max}_{\substack{X \in T \\\|X\| \underline{p}_{1}^{(M)}}}\left\|\left(\nabla_{X}^{\prime} \sigma\right)(X, X)\right\| .
$$

Let $\left\{X_{1}, X_{2}=J X_{1}\right\}$ be an orthonormal frame field near $p$ satisfying the condition (4.19) for the basis $\left\{E_{1}=E, E_{2}=J E\right\}$ at $p$. Then, we may easily see that $d=0$ (and hence $c^{2}+c=0$ ) at $p$. We may assume that $c=-1$ at $p$. We put

$$
\zeta=-\frac{d}{4} N\left(X_{1}, \sigma\left(X_{1}, X_{1}\right)\right)+\frac{c}{4} N\left(X_{2}, \sigma\left(X_{1}, X_{1}\right)\right) \quad \text { near } p .
$$

Then, taking account of (3.1), (3.7), (3.8), (4.2), (4.8), (4.9), (4.20) and (4.29), we get

$$
\begin{aligned}
\sigma_{p}^{4}\left(E_{1}, E_{1}, E_{1}, E_{1}\right) & =-\left(E_{2} d+a\right) \zeta_{p} \\
& =-2 G(p) \zeta_{p}=0 .
\end{aligned}
$$


Similarly, we get

$$
\sigma_{p}^{4}\left(E_{2}, E_{1}, E_{1}, E_{1}\right)=0 .
$$

Thus, from (4.31) and (4.32), taking account of (4.12) and the symmetricity of $\sigma_{p}^{4}$, we have finally $\sigma_{p}^{4}=0$, and hence the degree of $\phi$ is 3. This completes the proof of Theorem A. Next, we shall prove Theorem B. We assume that the Gaussian curvature $K$ of $M$ is constant and $K \neq 1$. From (4.1), we get $\|\sigma\|^{2}=2(1-K)$, and hence from (4.10) and (4.14)

$$
F=0 \quad \text { on } M=M^{\prime} \text {. }
$$

Thus, from (4.33) and Lemma 4.2, we get

$$
G=-\frac{3}{2} K \quad \text { on } M \text {. }
$$

From (4.34) and Lemma 4.3, it follows that $G(4 G+1)=0$. If $4 G+1=0$, then, from (4.34), we have $K=1 / 6$, and otherwise, we have $K=0$. This completes the proof of Theorem B.

Lastly, we shall prove Theorem $C$. We suppose that $M$ is compact and $M^{\prime} \neq \emptyset$. Then $\|\sigma\|$ takes its maximum at some point $p \in M^{\prime}$. Then, from (4.10), we have $F(p)=0$. Thus, from Lemmas 4.1 and 4.2 , we have

$$
0 \geqq(\Delta \log \|\sigma\|)(p) \geqq-\frac{1}{2}+3 K(p),
$$

and hence $K(p) \leqq 1 / 6$.

Thus, if $M$ is compact and $K>1 / 6$ on $M$, from (4.35), it follows that $M^{\prime}=0$, and hence the first half of Theorem $C$ is proved. The latter half of Theorem $C$ is immediately followed by using Lemmas 4.1 and 4.2, and Green's theorem. From Lemmas 4.2 and 4.3, taking account of Green's theorem and Gauss-Bonnet theorem, we have the following

THeOREM D. Assume that $M$ is compact and $K<1$ on $M$. If the function $G$ satisfies the inequality $-1 / 4 \leqq G \leqq 0$ on $M$, then $G=0$ or $-1 / 4$ on $M$, and furthermore $M$ is diffeomorphic to a 2-dimensional torus (resp. a 2-dimensional sphere) in the case where $G=0$ on $M$ (resp. $G=-1 / 4$ on $M$ ).

We remark that the equality $G=0$ (resp. $G=-1 / 4$ ) on $M^{\prime}$ is equivalent to

$$
\Delta \log (1-K)=6 K, \quad \text { on } M^{\prime}
$$

(resp. (4.37) $\Delta \log (1-K)=-1+6 K$ on $M^{\prime}$ ) 


\section{Some examples}

ExAMPLE 1. Let $M=\left\{x \in S^{6} ; x=x_{2} e_{2}+x_{4} e_{4}+x_{6} e_{6}\right\}$, and $c$ be the inclusion map from $M$ into $S^{6}$. Then, we may easily see that $(M, \iota)$ is a 2 -dimensional almost complex and totally geodesic submanifold of $S^{6}$.

EXAMPLE 2. Let $M=S_{1 / 6}^{2}=\left\{\left(y_{1}, y_{2}, y_{3}\right) \in \boldsymbol{R}^{3} ; y_{1}^{2}+y_{2}^{2}+y_{3}^{2}=6\right\}$ and $\phi_{0}$ be a $C^{\infty}$ map from $M$ into $S^{6}$ defined by

$$
\begin{aligned}
\phi_{0}\left(y_{1}, y_{2}, y_{3}\right) \\
=\left(\frac{\sqrt{6}}{72}\left(2 y_{1}^{3}-3 y_{1} y_{2}^{2}-3 y_{1} y_{3}^{2}\right)\right) e_{1}+\left(\frac{\sqrt{15}}{72}\left(3 y_{2}^{2} y_{3}-y_{3}^{3}\right)\right) e_{2} \\
+\left(\frac{\sqrt{15}}{72}\left(y_{2}^{3}-2 y_{2} y_{3}^{2}\right)\right) e_{3}+\left(\frac{1}{24}\left(4 y_{1}^{2} y_{2}-y_{2}^{3}-y_{2} y_{3}^{2}\right)\right) e_{4} \\
+\left(\frac{1}{24}\left(4 y_{1}^{2} y_{3}-y_{2}^{2} y_{3}-y_{3}^{3}\right)\right) e_{5}+\left(\frac{\sqrt{10}}{24}\left(y_{1} y_{2}^{2}-y_{1} y_{3}^{2}\right)\right) e_{6} \\
+\left(\frac{\sqrt{10}}{12} y_{1} y_{2} y_{3}\right) e_{7}, \quad \text { for } \quad\left(y_{1}, y_{2}, y_{3}\right) \in S_{1 / 6}^{2} .
\end{aligned}
$$

Then, we may easily check that $\left(S_{1 / 6}^{2}, \psi_{0}\right)$ is a 2-dimensional almost complex submanifold of $S^{6}$ and furthermore, any almost complex submanifold $\left(S_{1 / 6}^{2}, \phi\right)$ of $S^{6}$ is obtained by $\phi=\alpha \cdot \psi_{0}$ for some $\alpha \in G_{2}$.

EXAMPLE 3. Let $M=\boldsymbol{R}^{2}$ be a 2-dimensional Euclidean space with the canonical metric and $\phi$ be a $C^{\infty}$ map from $\boldsymbol{R}^{2}$ into $S^{6}$ defined by

$$
\begin{aligned}
\phi(u, v)= & \sqrt{\frac{2}{3}}\left(\cos \sqrt{\frac{1}{2}} u\right)\left(\left(\sin \sqrt{\frac{3}{2}} v\right) a_{1}-\left(\cos \sqrt{\frac{3}{2}} v\right) b_{1}\right) \\
& +\sqrt{\frac{2}{3}}\left(\sin \sqrt{\frac{1}{2}} u\right)\left(\left(\sin \sqrt{\frac{3}{2}} v\right) a_{2}-\left(\cos \sqrt{\frac{3}{2}} v\right) b_{2}\right) \\
& +\left(\sqrt{\frac{1}{3}} \cos \sqrt{2} u\right) a_{3}+\left(\sqrt{\frac{1}{3}} \sin \sqrt{2} u\right) b_{3},
\end{aligned}
$$

for $(u, v) \in \boldsymbol{R}^{2}$, where $a_{\imath}, b_{i} \in \mathcal{C}_{+}=\boldsymbol{R}^{7}$ such that $\left(a_{\imath}, a_{j}\right)=\delta_{i \jmath},\left(a_{\imath}, b_{j}\right)=0,\left(b_{i}, b_{j}\right)=\delta_{i \jmath}$, $1 \leqq \imath, j \leqq 3$, and

$$
\begin{aligned}
& a_{1} \times b_{1}=-b_{3}, \quad a_{3} \times a_{1}=b_{2}, \quad a_{3} \times b_{1}=-a_{2}, \\
& a_{2} \times b_{2}=b_{3}, \quad a_{1} \times a_{2}=b_{1} \times b_{2}=-a_{3} \times b_{3} .
\end{aligned}
$$

For example, $\left(a_{1}, a_{2}, a_{3}, b_{1}, b_{2}, b_{3}\right)=\left(e_{3},-e_{2}, e_{5},-e_{7}, e_{6}, e_{4}\right)$ satisfies the relations in (5.2). We may easily check that $\left(\boldsymbol{R}^{2}, \psi\right)$ is a 2 -dimensional almost complex submanifold of $S^{6}$. 
The above immersion $\psi$ induces an immersion $\Psi: T^{2}=\boldsymbol{R}^{2} / \Gamma \rightarrow S^{6}$ in the natural way, where $\Gamma$ denotes the lattice group in $\boldsymbol{R}^{2}$ generated by $\{2 \sqrt{ } 2 \pi(1,0)$, $\left.2 \sqrt{\frac{2}{3}} \pi(0,1)\right\}$

\section{REFERENCES}

[1] E. CAlaBi, Minimal immersion of surfaces in Euclidean spheres, J. Diff. Geom., 1 (1967), 111-125.

[2] M.P. Do CARmo And N.R. WAllach, Representations of compact groups and minimal immersions into spheres, J. Diff. Geom., 4 (1970), 91-104.

[3] T. Fukami and S. Ishinara, Almost Hermitian structure on $S^{6}$, Tōhoku Math. J., 7 (1955), 151-156.

[4] A. GRAY, Almost complex submanifolds of six sphere, Proc. Amer. Math. Soc., 20 (1969), 277-279.

[5] K. Kenmotsu, On minimal immersions of $\boldsymbol{R}^{2}$ into $S^{N}$, J. Math. Soc. Japan., 28 (1976), 182-191.

[6] K. Kenmotsu, The classification of minimal surfaces with constant Gaussian curvature in a space form, to appear.

[ 7 ] M. Matsumoto, On 6-dimensional almost Tachibana spaces, Tensor N.S., 23 (1972), 250-252.

[8] H. NAKAGAWA, On a certain minimal immersion of a Riemannian manifold into a sphere, Kōdai Math. J., 3 (1980), 321-340.

[9] K. Takamatsu, Some properties of 6-dimensional $K$-spaces, Kōdai Math. Sem. Rep., 23 (1971), 215-232.

[10] S. Sawaki and K. Sekigawa, Almost Hermitian manifold with constant holomorphic sectional curvature, J. Diff. Geom., 9 (1974), 123-134.

[11] S. SAWAKI AND K. SEKIGAWA, $\sigma$-hypersurfaces in a locally symmetric almost Hermitian manifolds, Tōhoku Math. J., 30 (1978), 543-551.

[12] L. Vanhecke, On immersions with trivial normal connections in some almost Hermitian manifolds, Rendiconti di Matematica (I) Vol. 10, Serie VI., (1977), 75-86.

[13] K. Yano, Differential geometry on complex and almost complex spaces, Pergamon Press. 1965.

NiIgATA University

NiIGATA, JAPAN 